\title{
When Should We Start the Lockdown and How Long Should It Be?
}

\author{
Yasunori Fujita \\ Keio University, Tokyo, Japan \\ Email: yfujita@econ.keio.ac.jp
}

How to cite this paper: Fujita, Y. (2020). When Should We Start the Lockdown and How Long Should It Be? Modern Economy, 11, 1007-1011.

https://doi.org/10.4236/me.2020.115075

Received: April 7, 2020

Accepted: May 6, 2020

Published: May 9, 2020

Copyright $\odot 2020$ by author(s) and Scientific Research Publishing Inc. This work is licensed under the Creative Commons Attribution International License (CC BY 4.0).

http://creativecommons.org/licenses/by/4.0/

\begin{abstract}
As the new coronavirus spreads around the world, some countries have already gone into the lockdown; other countries are ready for it. Given this fact, the present paper investigates the optimal start time of the lockdown and its length, by constructing a stochastic model based on optimal stopping theory. We reveal that if the lockdown does not damage the economy so much, the government should start the lockdown earlier and make it longer. We also show that if uncertainty increases, the government should postpone the start of the lockdown, keeping its length unchanged.
\end{abstract}

\section{Keywords}

Coronavirus, Lockdown, Optimal Stopping Theory, Value of Waiting

\section{Introduction}

As the new coronavirus spreads around the world, many governments in the world have been taking measures to prevent the deterioration of the economy, such as requests to only go outside for essential things, interest free cash grants to small businesses, Value Added Tax payments by companies deferred for several months and so on.

In Japan, for example, focus is on encouraging people to avoid an overlap of what is called "Three Cs", crowded spaces with insufficient ventilation, crowded conditions with people and conversations in a short distance. Now, as things are getting even worse, some countries, such as Denmark, Germany etc., have already gone into the lockdown; other countries, especially Japan, are ready for it.

In the present paper, we attempt to reveal when the government should start the lockdown and how long it should be. The model we construct is a stochastic one; the theory we make use of is the optimal stopping theory, which deals with 
the problem of choosing the optimal time to take a particular action in a stochastic environment, in order to maximize an expected profit or minimize an expected cost. One of the most distinguished works is McDonald and Siegel (1986), which demonstrated the value of waiting, followed by Dixit (1989), Farzin, Huisman and Kort (1988), Fujita $(2007,2018)$ and so on. The present paper, by pushing forward these studies, shows the usefulness of the optimal stopping theory in tackling the current coronavirus problem.

Structure of this paper is as follows. After constructing a basic stochastic model in Section 2, Section 3 formulates the objective function of the government. Based on these analyses, in Section 4 we reveal when the government should start the lockdown and how long it should be. Concluding remarks are made in Section 5.

\section{Basic Model}

Let us consider an inter-temporal economy where time passes continuously and the time horizon is infinite.

We assume that the total number of people in the economy is $N$ and the number of people who are infected with the new coronavirus in period $t$ is $n(t)$. We also assume the initial value of $n(t)$ is 0 and the rate of newly infected people to the total number of people in the economy is $\alpha$. Under these assumptions, motion of $n(t)$ is expressed as

$$
\frac{\mathrm{d} n}{\mathrm{~d} t}=\alpha(t)(N-n(t))
$$

and by solving this differential equation, we have the number of infected people in period $t, n(t)$, as

$$
n(t)=N\left(1-\mathrm{e}^{-\alpha t}\right)
$$

Throughout this paper, we assume that $\alpha(t)$ follows the geometric Brownian motion of Equation (3).

$$
\mathrm{d} \alpha=\mu \alpha \mathrm{d} t+\sigma \alpha \mathrm{d} z
$$

with initial value of $\alpha$ being $\alpha_{0}$. $\mu$ and $\sigma$ are parameters of drift and volatility, with both $\mu$ and $\sigma$ being positive constants. Larger $\mu$ means that $\alpha(t)$ increases more quickly; larger $\sigma$ means that the growth of $\alpha(t)$ is more uncertain. $\mathrm{d} z$ is Wiener process that expresses random movement, which has several real-world applications such as stock market fluctuations, exchange rate fluctuations and so on.

We assume that the amount of GDP decreases as the number of infected people, $n(t)$, increases. In order to simplify the analysis, letting $Y$ denote the amount of GDP with no infected people, we specify the amount of GDP in period $t, Y(t)$, as

$$
Y(t)=Y-\theta n(t)
$$


where $\theta$ is a positive constant. By substituting (2) into (4), we have the GDP in period $t$ as

$$
Y(t)=Y-\theta N+\theta N \mathrm{e}^{-\alpha(t) t}
$$

as a function of $\alpha(t)$.

\section{Formulation of the Objective Function of the Government}

The government in the present paper is assumed to start the lockdown if $\alpha(t)$ reaches $\alpha^{*}$, by incurring the cost $K$, at the moment it starts the lockdown. We also assume that if the government puts the entire country on lockdown, GDP drops to a constant value $m$, and that longer lockdown removes more coronavirus, to increase the GDP after the lift of the lockdown. In the following, in order to simplify the analysis, we specify the GDP after the lift of the lockdown to be constant at $Y-\frac{C}{S}$, where $c$ is a positive parameter and $S$ is the length of the lockdown, which the government determines.

Letting $r$ denote the discount rate, and assuming the government to maximize sum of the expected present value of GDP minus expected present value of the cost $K$, we can express the government's objective function $V$ as follows.

$$
\begin{aligned}
V= & \int_{0}^{\tau} \mathrm{e}^{-r t}\left(Y-\theta N+\theta N \mathrm{e}^{-\alpha(t) t}\right) \mathrm{d} t+\int_{\tau}^{\tau+S} \mathrm{e}^{-r t} m \mathrm{~d} t \\
& +\int_{\tau+S}^{\infty} \mathrm{e}^{-r t}\left(Y-\frac{C}{S}\right) \mathrm{d} t-\mathrm{e}^{-r \tau} K
\end{aligned}
$$

We assume that the government maximizes $V$ with respect to $S$ and $\alpha^{*}$.

In order to simplify (6), let us calculate the expected present value of one unit of GDP at the moment when $\alpha(t)$ whose initial value is $\alpha_{0}$ reaches $\alpha^{*}$.

Letting $G\left(\alpha_{0}\right)$ denote this value, we can express the general solution to $G\left(\alpha_{0}\right)$ as

$$
G\left(\alpha_{0}\right)=A\left(\alpha_{0}\right)^{\beta_{1}}+B\left(\alpha_{0}\right)^{\beta_{2}}
$$

where $\beta_{1}<0$ and $\beta_{2}>0$ are solutions to $\frac{1}{2} \sigma^{2} x(x-1)-r=0$. Since $G\left(\alpha_{0}\right)$ satisfies $G(0)=0$ and $G\left(\alpha^{*}\right)=1$, it follows that $A=0$ and $B=\frac{1}{\alpha^{* \beta_{2}}}$. Since substituting these equations into (2) yields $G\left(\alpha_{0}\right)=\left(\frac{\alpha_{0}}{\alpha^{*}}\right)^{\beta_{2}}$, we obtain

$$
G(\alpha)=\left(\frac{\alpha_{0}}{\alpha^{*}}\right)^{\beta}
$$

by letting $\beta$ denote $\beta_{2}$. Thus, we can rewrite (6) as

$$
\begin{aligned}
V= & \frac{1}{r}(Y-\theta N)\left(1-\left(\frac{\alpha_{0}}{\alpha^{*}}\right)^{\beta}\right)+\frac{1}{\mu+r} \theta N\left(1-\left(\frac{\alpha_{0}}{\alpha^{*}}\right)^{\beta} \alpha^{*}\right) \\
& -\left(\frac{\alpha_{0}}{\alpha^{*}}\right)^{\beta} \frac{m}{r}\left(1-\mathrm{e}^{-r S}\right)+\left(\frac{\alpha_{0}}{\alpha^{*}}\right)^{\beta} \mathrm{e}^{-r S} \frac{1}{r}\left(Y-\frac{c}{S}\right)-\left(\frac{\alpha_{0}}{\alpha^{*}}\right)^{\beta} \mathrm{e}^{-r \tau} K
\end{aligned}
$$

by making use of (8). 


\section{Optimal Start Time of the Lockdown and Its Length}

Based on the above analysis, in this section we derive the optimal start time of the lockdown and its length.

Since the government in the present paper maximizes $V$ with respect to $S$ and $\alpha^{*}$, the first order conditions for optimization are $\frac{\partial V}{\partial S}=0$ and $\frac{\partial V}{\partial \alpha^{*}}=0$. First of all, from $\frac{\partial V}{\partial S}=0$ we have

$$
(Y-m) r S^{2}-r c S-c=0,
$$

which yields the optimal value of $S$ as

$$
S=\frac{r c+\sqrt{r^{2} c^{2}+4 c(Y-m)}}{2(Y-m)}
$$

Similarly, from $\frac{\partial V}{\partial \alpha^{*}}=0$ we have

$$
\begin{aligned}
& \frac{1}{r}(Y-\theta N) \beta \alpha_{0}^{\beta} \alpha^{*-\beta-1}-\frac{\theta N}{\mu+r}(\beta+1) \alpha_{0}^{-\beta} \alpha^{* \beta}-\beta \alpha_{0}^{\beta} \alpha^{*-\beta-1} \frac{m}{r}\left(1-\mathrm{e}^{-r S}\right) \\
& -\beta \alpha_{0}^{\beta} \alpha^{*-\beta-1} \frac{1}{r}\left(Y-\frac{C}{S}\right)+\beta \alpha_{0}^{\beta} \alpha^{*-\beta-1} K=0
\end{aligned}
$$

which yields the optimal value of $\alpha^{*}$ as

$$
\alpha^{*}=\left[\frac{\mu+r}{\theta N} \frac{\beta}{\beta-1} \alpha_{0}^{2 \beta}\left[\frac{1}{r}\left\{\frac{c}{S}-\theta N-m\left(1-\mathrm{e}^{-r S}\right)\right\}+K\right]\right]^{\frac{1}{2 \beta+1}}
$$

where $S$ is the value determined in (10).

From (10) and (11), we have the following proposition.

Proposition 1: If the lockdown does not damage the economy so much, the government should make the length of the lockdown longer start it earlier.

Proof of Proposition 1:

From (10) and (11), we have $\frac{\partial S}{\partial m}>0$ and $\frac{\partial \alpha^{*}}{\partial m}<0$, which means that larger $m$ increases $S$ and reduces $\alpha^{*}$, respectively. $m$ is the amount of GDP after the lockdown, larger $S$ means longer period of the lockdown and smaller $\alpha^{\star}$ means earlier start of the lockdown. Thus, we have the proposition 1.

Similarly, we have the following proposition that reveals the relationship between uncertainty and the lockdown.

Proposition 2: If uncertainty increases, the government should postpone the start of the lockdown, keeping its length unchanged.

Proof of Proposition 2:

Since $\beta$ is a positive solution of $\frac{1}{2} \sigma^{2} x(x-1)-r=0$, we have $\frac{\partial \beta}{\partial \sigma}<0$, which means that increase in $\sigma$ reduces $\beta$; from (10) and (11), $\frac{\partial S}{\partial \beta}=0$ and $\frac{\partial \alpha^{*}}{\partial \beta}<0$. By combining $\frac{\partial \beta}{\partial \sigma}<0$ and $\frac{\partial S}{\partial \beta}=0$, we have $\frac{\partial S}{\partial \sigma}=0 ; \frac{\partial \alpha^{*}}{\partial \sigma}<0$ and 
$\frac{\partial \alpha^{*}}{\partial \beta}<0$, we have $\frac{\partial \alpha^{*}}{\partial \sigma}>0$. Since larger $\alpha^{*}$ means postponement of the start of the lockdown, we have the proposition 2.

This proposition is consistent with the postponing phenomenon resulting from the value of waiting, which is often seen in the standard literatures on the optimal stopping theory.

\section{Conclusion}

We began this research wishing to contribute to the progress of the studies on the new coronavirus problems. We revealed that if the lockdown does not damage the economy so much, the government should start the lockdown earlier and make it longer. We also showed that if uncertainty increases, the government should postpone the start of the lockdown, keeping its length unchanged. This result is consistent with the postponing phenomenon resulting from the value of waiting, which is often seen in the standard literatures on the optimal stopping theory such as Dixit and Pindyck (1994).

In the present paper, for the sake of simplicity, we made simplifying assumptions on the relationship between GDP and the number of infected people, motion of the number of those who are infected with the new coronavirus and so on. Thus, it is necessary to examine the robustness of our results in a more general framework. It is also important to find the way of sustaining both the economy and the medical system. It is of interest to examine how much should government compensate firms for suspension of their businesses. We will undertake such analyses in future research.

\section{Conflicts of Interest}

The author declares no conflicts of interest regarding the publication of this paper.

\section{References}

Dixit, A. K. (1989). Hysteresis, Import Penetration, and Exchange Rate Pass Through. Quarterly Journal of Economics, 104, 205-228. https://doi.org/10.2307/2937845

Dixit, A. K., \& Pindyck, P. (1994). Investment under Uncertainty. Princeton, New Jersey: Princeton University Press. https://doi.org/10.1515/9781400830176

Farzin, Y. H., Huisman, K. J. M., \& Kort, P. M. (1988). Optimal Timing of Technology Adoption. Journal of Economic Dynamics and Control, 22, 779-799. https://doi.org/10.1016/S0165-1889(97)00097-3

Fujita, Y. (2007). Toward a New Modeling of International Economics: An Attempt to Reformulate an International Trade Model Based on Real Option Theory. Physica A: Statistical Mechanics and its Applications, 383, 507-512.

https://doi.org/10.1016/j.physa.2007.04.041

Fujita, Y. (2018). Counter Cowbell Effect in a Stochastic Market: Does Subsidy Really Induce Foreign Direct Investment? Modern Economy, 9, 790-795. https://doi.org/10.4236/me.2018.94052

McDonald, R., \& Siegel, D. (1986). The Value of Waiting to Invest. The Quarterly Journal of Economics, 101, 707-727. https://doi.org/10.2307/1884175 